\title{
La importancia de la educación motriz en el proceso de enseñanza de la lecto - escritura en niños y niñas del nivel preprimaria y de primero
}

\section{The importance of motor education in the process of teaching reading and writing in boys and girls at the preprimary and first level}

\author{
Zila Isabel Esteves Fajardo \\ Universidad de Guayaquil, Ecuador \\ Verónica Narcisa Toala Santana \\ Elizabeth Eunice Poveda Gurumendi \\ Colegio Emblemático Guayaquil, Ecuador \\ Mercedes Quiñonez \\ Universidad de Guayaquil, Ecuador
}

Autor para correspondencia: zilaisabelesteves@hotmail.es, Norma.garcesg@ug.edu.ec, veritont@hotmail.com, lizlpz30@yahoo.com

Fecha de recepción: 25 de Febrero de 2018 - Fecha de aceptación: 15 de Julio de 2018

Resumen: La lectura y la escritura no sólo podemos definirlas como meras herramientas académicas, si no como unos instrumentos fundamentales para el crecimiento personal y social de los individuos. En el presente trabajo realizaremos un análisis de la lectura y la escritura en la etapa de Educación Primaria, los diferentes métodos para trabajarlas, dentro y fuera de la escuela, además de las diferentes actividades a desarrollar para alcanzar los objetivos propuestos y la consecución de las competencias básicas en todas las áreas de la etapa. Ya que esto es un trabajo no solo para los maestros sino también para los padres para que ayuden a un mejor desarrollo y desenvolvimiento de los niños y así tengan confianza y seguridad en lo que hacen y así pueden lograr muchas cosas más. Ya que es el resultado de los logros alcanzados por el niño en el dominio de los movimientos finos de la mano.

Palabras Claves: lectura; escritura; aprendizaje; enseñanza; métodos; estrategias

Abstract: Reading and writing cannot only be defined as mere academic tools, but as fundamental instruments for personal and social growth of individuals. In the present work we will carry out an analysis of reading and writing in the stage of Primary Education, the different methods to work them, inside and outside the school, in addition to the different activities to be developed to reach the proposed objectives and the attainment of the Basic competences in all areas of the stage. Since this is a job not only for the teachers but also for the parents so that they help to a better development and development of the children and thus they have confidence and security in what they do and thus they can achieve many more things. Since it is the result of the achievements of the child in the domain of the fine movements of the hand.

Key words: reading; writing; learning; teaching; methods; strategies 


\section{Introducción}

La lectoescritura constituye un pilar básico en la vida de todo ser humano. No solo se trata de una herramienta indispensable para acceder a los objetivos y contenidos educativos si no que es uno de los principales vehículos de la cultura existente. Tomando como referencia (Boletín Oficial Español , 2006 ) y siguiendo la Ley Orgánica 2/2006 de 3 de mayo de Educación, el presente trabajo reflejará un estudio de la lectoescritura en la etapa de Educación Primaria, analizando los diferentes factores que influyen en el proceso de enseñanza-aprendizaje del mismo, como pueden ser: los aspectos que intervienen en la adquisición de la lectoescritura, el fomento de la lectura, así como los elementos que favorecen u obstaculizan dichos procesos, teniendo en cuenta las dimensiones ambientales (ámbito escolar, el medio, la familia...)

La psicomotricidad, plantea que la psicomotricidad no puede reducirse exclusivamente a lo motriz, puesto que aspectos como la sensación, la relación, la comunicación, la afectividad, el lenguaje o la integración inciden en el proceso de desarrollo o del tratamiento psicomotor de manera importante y siempre ligada al movimiento. El dominio corporal es la capacidad de dominar las diferentes partes del cuerpo: extremidades superiores e inferiores, tronco, de hacerlas mover siguiendo la voluntad o realizando una consigna determinada, permitiendo tan solo un movimiento de desplazamiento sino también una sincronización de movimientos, superando las dificultades que los objetos y el espacio o el terreno impongan, llevándolo a cabo de una manera armónica, precisa, sin rigideces ni brusquedades.

Citado por (Miganjos, 2005) García, en el año 2004, logró establecer en la investigación realizada sobre la Influencia de la Psicomotricidad Global en el Desarrollo de la Psicomotricidad Fina, que una buena metodología para la aplicación de la psicomotricidad global y fina permite al alumno desarrollarse y adaptarse mejor no solo en la vida educativa sino en la vida social familiar y cultural dándole lugar a la madurez emocional, intelectual y de su personalidad, el estudio fue realizado en la Escuela Urbana de Párvulos Edelmira Mauricio, del municipio de Huehuetenango.

En los últimos años se ha argumentado que el aprendizaje precoz de la lecto-escritura podría ocasionar daños en el sistema nervioso del niño o la niña, sin embargo, actualmente se ha demostrado que esto no es así y que se puede enseñar de forma adecuada a cualquier niño y en cualquier etapa.

Lo que realmente es importante para dicho aprendizaje, más que la edad cronológica del sujeto, son las características que se deben poseer para llevar a cabo tal aprendizaje. Las características o condiciones previas para el aprendizaje de la lecto-escritura son: a. Neurofisiológicas: como pueden ser el carecer de dificultades sensoriales que impidan dicho aprendizaje, contar con el suficiente desarrollo muscular sin trastornos motrices, poseer la capacidad para el manejo de instrumentos, tener estructurada la imagen corporal y dominar la coordinación digito - manual entre otras. b. Cognitivas: como pueden ser carecer de trastornos severos en el funcionamiento de los procesos cognitivos y haber logrado un buen desarrollo perceptivo. c. Socio emocional: dentro de las que estarían la confianza en sí mismo y la madurez emocional. Además, no se debe olvidar que el proceso debe desarrollarse en ambientes favorecedores de comunicación y potenciando siempre la autoestima. Por otro lado, la enseñanza debe ser lo más funcional posible y haciendo uso de materiales de enseñanza adecuados. Antes 
de iniciarse las actividades para el aprendizaje de este proceso, es necesario, hacer un diagnóstico o evaluación con el objetivo de obtener información sobre diferentes aspectos del alumno o la alumna, los cuales pueden ir desde historia escolar, hasta la evolución neurológica, psíquica y emocional/afectiva.

Durante la implementación del programa se deben de proponer dos tipos de actividades:

a) Ejercicios no gráficos para el entrenamiento de los procesos cognitivos implicados en el acto gráfico. Serán ejercicios de relajación, de psicomotricidad, de percepción visual, coordinación viso-motora, orientación espacio-temporal, atención, equilibrio, esquema corporal, percepción sensorio motriz, lateralidad, nociones de espacio y tiempo.

b) Los ejercicios gráficos que son aquellos en que se ejercita la escritura propiamente dicha como pueden ser: la realización de trazos rectos, curvas, líneas quebradas, onduladas, bucles grandes y pequeños, giros con desplazamiento hacia la derecha y realización de dibujos. Existen numerosas corrientes con respecto al campo de la psicomotricidad, las cuales responden a diversas teorías y concepciones, las cuales pueden privilegiar distintas posibilidades y desarrollarse en distintos ámbitos sociales pueden utilizarse diferentes fines y métodos para su implementación, cada una de ellas puede ponerse en práctica con situaciones particulares. Las teorías de la psicomotricidad surgieron en Europa y se expandieron por la mayoría de países desarrollados en América Canadá y Estados Unidos de América fueron los primeros posteriormente fueron asimilados por los países latinoamericanos. Al inicio se asimilaron bien, aunque perdieron su riqueza teórico conceptual, como sucede comúnmente solo los aspectos empíricos y técnicos, se difundieron únicamente las dimensiones más superficiales y generales, las que fueron repitiéndose mecánicamente, sin adaptación, defendiendo solamente los aspectos dogmáticos.

El desarrollo de la motricidad fina, es el resultado de los logros alcanzados por el niño en el dominio de los movimientos finos de la mano, de los pies, la coordinación óculo-manual, óculo pedal, la orientación espacial y la lateralidad, bajo la influencia del adulto, quien de manera intencionada o no, le va mostrando los modos de conducta motriz socialmente establecidos, que le permiten al niño desarrollar su independencia, realizar acciones cada vez más complejas y perfeccionarlas. Se consideran la edad preescolar el momento en el cual la estimulación constituye un determinante del desarrollo por una serie de factores que lo proporcionan: el acelerado crecimiento, el desarrollo de los sistemas sensoriales, del sistema osteomioarticular (la flexibilidad de los músculos y tendones, calcificación de los huesos, presentándose en la mano, en el pie, en la musculatura de la cara diferentes etapas de maduración), la facultad de establecer innumerables conexiones nerviosas temporales, así como el proceso de mielogénesis, que constituyen la base fisiológica que permiten configurar las condiciones para el aprendizaje de las diferentes acciones.

El desarrollo psicomotor es muy importante para el niño y la niña, porque la progresiva maduración de la neuro musculatura en la edad preescolar permite realizar con destreza, las diversas actividades motoras; el aprendizaje desempeña un papel esencial en el mejoramiento de las mismas, siempre que haya un adecuado desarrollo neuromuscular. El rendimiento motor varía con la motivación, la emoción y los apoyos físicos que el niño tenga en la casa y en la escuela; el 
movimiento provoca en el niño una satisfacción natural, y genera un desarrollo básico para conseguir satisfacción y seguridad emocional. La educación del movimiento procura la mejor utilización de las capacidades psíquicas del niño, es decir, permite la interacción de las diversas funciones motrices y psíquicas.

La búsqueda de antecedentes en los diversos centros de documentación, bibliotecas e Internet, conllevo a identificar una serie de trabajos que sirven de guía para este trabajo de investigación. Así, según (Miganjos, 2005) manifiesta que datos aportados en 1994 por investigaciones realizadas por Cornegie Corporation en Estados Unidos, revelan que el medio ambiente, no sólo afecta el número de células cerebrales, las posibilidades de sinapsis entre ellas y la manera como estas conexiones se establecen, sino que esa influencia temprana del medio exterior deja huellas definitivas en la psiquis humana, y que su falta causa daños irreversibles en el individuo.

Para la Universidad de Valladolid en Soria (Lucas, 2014) realizó un estudio sobre la lectoescritura en la época de Educación primaria, llegando a establecer que: Para que se produzca la comprensión auditiva es necesario que se produzca una relación de imagen visual a nivel de lóbulo occipital y el habla en el lóbulo frontal, además de representarse en sonido mediante una letra que es el grafema. Además hace énfasis que algunas de las dificultades en la adquisición de la lectura se deben a un aumento en la latencia de la maduración del cerebro, lo que lleva a un retraso en la discriminación auditiva y el desarrollo fonológico, retrasando después las habilidades lingüístico- conceptuales, como son la semántica y la sintaxis.

(Miganjos, 2005), al referirse a la lectoescritura y su relación con la motricidad considera que un adecuado desarrollo perceptivo-motor es requisito preliminar para el aprendizaje de la lectura. El mismo autor aludiendo a Piaget menciona que el dinamismo motor y los concretos aprendizajes motrices están estrechamente ligados a la actividad mental infantil. También hace alusión a lo que Espenschade en la década del 70, presentaba una serie de concepciones pedagógicas que trajo consigo el reconocimiento de la totalidad del niño, la autora introdujo muchos tipos de actividad física en la escuela a medida que se les brindaba a los alumnos la oportunidad de aprender mediante la acción. Considera que el aprendizaje de los niños pequeños es un considerable media de índole sensorio motriz.

La acción y la conceptualización de imágenes son los que van a lograr establecer la verdadera relación grafema y lo que se lee en ese mundo interno que va a formar la idea, así Kephart, citado por (Miganjos, 2005), considera que, para que se dé el aprendizaje de una manera efectiva, la percepción y el movimiento, considerado este último como el medio a partir del cual un individuo adquiere conciencia del mundo y de sí mismo, debe marchar simultáneamente, de modo que el proceso de aprendizaje lleva una gran variedad de experiencias sensoriales y oportunidades de movimiento.

El gran abismo de la escritura sigue siendo una telearaña epistemológica que no debe romperse: sonido- letra y significado de ambos elementos o lo que Descartes llamaría en su momento la res cogitans del proceso. Sí a ese triángulo se le agrega el movimiento de lo que se ve, o se escucha que lleva a la formalización de las ideas, lo que se escriba con significado aunque sea poco formará parte de la conciencia fonoauditiva del niño, en la que percepción y 
movimiento funcionan como una sola cosa. El movimiento es un elemento básico en el aprendizaje pues promueve el eficiente proceso cognoscitivo. La psicomotricidad es una actividad que facilita el desarrollo integral del niño.

Las áreas que comprende la psicomotricidad son:

- Esquema corporal.

- Lateralidad.

- Equilibrio: estático, dinámico de los objetos.

- Motricidad gruesa.

- Motricidad fina: coordinación ojo-pie, coordinación ojo-mano, coordinación ocular.

- Ritmo.

- Disociación.

- Relajación.

Esa psicomotricidad se debe unir ahora a un mundo simbólico, al de la letra y su significado. ¿Qué valor tiene una vocal? ¿Qué les da valor semántico a las vocales? ¿Es el niño consciente del valor de las vocales? El año 2012 en México se hizo una investigación para la Universidad de México en Zamora Michoaclán, en la que la Universidad Pedagógica Nacional de la mano de Gabriela Sepúlveda López escribía: "La psicomotricidad fina y su importancia para la adquisición de la lectoescritura", en la que la autora hace énfasis en la coordinación manual, y expresa (Sepúlveda, 2012) que el hombre no tiene movimientos limitados y fijos, sino que puede dirigirlos y mantenerlos bajo control, las manos y parte visible de la inteligencia " el hombre puede decirse que toma posesión del ambiente con sus manos.", en la que la actividad manipulativa o kinestésica es muy importante para obtener información, Cratty, plantea que a medida que los niños establecen contacto con objetos por medio de sus manos, pasan por tres fases generales: 1) contacto simple; 2) presión palmar rudimentaria e inspección; 3) formación de copias motrices exactas de los objetos, mediante su inspección táctil precisas. Por ello en esta coordinación se debe llegar a tener el control de movimiento de los dedos, manos y muñecas, mediante actividades o ejercicios continuos y completos que implica dominio de los músculos de la muñeca y de los dedos en la ejecución de movimientos finos, adquiridos sólo a base de actividades encaminadas a dar soltura a las manos y a los dedos.

Para (Miganjos, 2005) al hacer el análisis de Muchielli quien a fines de 1979 escribe que gran cantidad de estudios sobre la dislexia, sostiene, incluso, que la educación motriz o psicomotriz no debe ser sólo una parte del programa escolar o reducirla a unas sesiones determinadas, sino que debe estar en la base de cualquier actividad y de cualquier aprendizaje en estas edades, porque la escritura, actividad convencional y codificada, es el fruto de una adquisición. Está constituida por signos que por su forma no tienen ningún valor directamente simbólico. Es simbólica en relación con la significación que el sujeto adquiere por el aprendizaje, a la vez, esta modalidad es gnosis(reconocimiento) - praxis (ejecución) y lenguaje, producto de una actividad psicomotriz, cognitiva sumamente complicada en la cual intervienen varios factores como son, entre otros, la maduración general del sistema nervioso; el desarrollo psicomotor general, sobre todo en lo concerniente al sostén tónico, a la coordinación de 
movimientos y de las actividades minuciosas de los dedos y las mano; y la adecuada estructuración de las habilidades comunicativas y el manejo del espacio.

Se debe dar gran importancia al desarrollo de la coordinación manual que considera ligada íntimamente a las capacidades intelectuales del niño. Buenos programas de experiencias motrices en niños de preescolar y educación básica pueden mejorar las tareas escolares, por las siguientes razones:

1) Las habilidades físicas desarrolladas en el juego y en periodos más estructurados de educación física, mejorarán los sentimientos generales de eficiencia en el niño.

2) Las destrezas motrices creativas fomentadas por medio de diversas formas de expresión artística y de juegos motores en los que se estimula un comportamiento selectivo en los niños, probablemente desembocarán en una participación más activa y cualitativa en todos los aspectos de interacción social que prevalecen en las escuelas elementales.

3) Los niños activos, si están demasiados tiempos quietos y encerrados, seguramente aprenderán con menos entusiasmo las tareas de clase. Los investigadores sugieren que tener inmóviles durante periodos prolongados a niños con buena condición física, que necesitan desplegar gran actividad, puede estar contraindicado en las escuelas elementales.

4) El movimiento parece ser una necesidad inherente a los niños, que al ser reprimido puede conducir a comportamientos indeseables.

Gran parte del fracaso escolar pueda ser causado por la "prematura intelectualización" de los aprendizajes escolares, propone una mayor participación de la dimensión corporal en la educación básica.

El control que puede tener el niño en sus movimientos, o sea, poder seleccionarlos e inhibirlos, depende de la evolución del niño con su cuerpo, siendo necesario tomar en cuenta la atracción que ejercen sobre él las personas de mayor edad y las condiciones sociales en las que se desenvuelve y de esto dependerá sus hábitos, habilidades, costumbres, la imitación y la repetición que son las formas en que el niño aprende, comprendiendo el dominio dinámico el cual es la capacidad de dominar las diferentes partes del cuerpo: extremidades superiores, inferiores y tronco, de hacerlas mover siguiendo la voluntad o realizando una consigna determinada, permitiendo no solo el movimiento y desplazamiento sino también una sincronización entre las dificultades de los objetos, el espacio y el terreno de búsqueda.

\section{El Desarrollo motor}

Segun (Wallon, 1879- 1962), nos manifiesta en su investigacion que;

La psicogénesis se produce en la interacción entre las posibilidades orgánicas y psicológicas del niño en cada momento evolutivo y el medio físico y social, este último de mayor importancia. En las relaciones del niño con el medio se produce una alternancia de modificaciones recíprocas, el niño es afectado por el medio y el medio es modificado por el niño (Reacción circular). Este concepto de reacción circular que posteriormente popularizara aún más Piaget, había sido formulado anteriormente por James Mark Baldwin.

Se refleja a través de la capacidad de movimiento, depende de dos factores básicos:

A. La maduración del sistema nervioso o mielinización de las fibras nerviosas, la cual sigue dos leyes: la cefalocaudal (de la cabeza al glúteo) y la próximo-distante (del eje a las 
extremidades). Las cuales explican por qué el movimiento en un principio es tosco, globo y brusco. En los primeros años la realización de los movimientos precisos depende de la maduración.

B. La evaluación del tono, sirve de fondo sobre el cual surgen las contracciones musculares y los movimientos; por tanto, es responsable de toda acción corporal y, además, es el factor que permite el equilibrio necesario para efectuar diferentes posiciones.

La calidad del tono se expresa a través de la extensibilidad muscular, la motilidad y el relajamiento muscular llamado, según la manifestación tónica, de rigidez con hipertonía o de relajamiento con hipotonía.

\section{El desarrollo motor se divide en tres fases:}

- Primera fase: del nacimiento a los 6 meses, se caracteriza por la dependencia total de la actividad refleja, especialmente el de la succión. A los tres meses desaparece debido a los estímulos externos que provocan el ejercicio y establecen la posibilidad de otras acciones y el inicio de movimientos voluntarios.

- Segunda fase: de los 6 meses a los 4 años, se caracteriza por la organización de las nuevas posibilidades del movimiento. Existe una movilidad más grande que se integra con la elaboración del espacio y el tiempo. Está ligada con el tono y la maduración.

- Tercera fase: de los 4 a los 7 años, corresponde a la automatización de las posibilidades motrices que forman la base necesaria para futuras adquisiciones.

La Psicomotricidad tiene como objetivo favorecer la relación entre el niño y su medio, proponiendo actividades relacionadas con 5 principios básicos que son:

1. La percepción: que es la manera en que el niño toma conciencia del medio ambiente, esta puede ser innata ya que puede percibir sensaciones desde los primeros meses de vida y aprendida porque logra desarrollarla según los estímulos que recibe del exterior. La percepción sensorio motriz, es el conjunto de estimulaciones visuales, auditivas y táctiles, es muy importante en el proceso escolar ya que aprende a discriminar formas, sonidos y colores.

2. Esquema Corporal: que se refiere al concepto que tiene una persona de su cuerpo y de sí mismo. Es fundamental para la elaboración de la personalidad y determinante en el proceso de aprendizaje.

3. Lateralidad: es el resultado de una predominancia motriz en el cerebro, se presenta en los segmentos corporales derecho e izquierdo, tanto a nivel ocular, como de las manos y los pies. Depende de dos factores: el desarrollo neurológico y de las influencias culturales que recibe. Se pueden distinguir dos tipos de lateralidad: a. Lateralidad de utilización: prevalencia manual de las actividades corrientes o sociales. b. Lateralidad espontánea: se manifiesta por una lateralidad tónica, en el lado dominante hay una tensión mayor. Los problemas en este desarrollo tienen consecuencias en la vida cotidiana del niño y repercuten en el aprendizaje escolar, especialmente en la lectura y escritura.

4. Elaboración del Espacio: la construcción del espacio se hace paralelamente a la elaboración del esquema corporal, ambos dependen de la evolución de los movimientos. El espacio se vive según las aferencias táctiles, auditivas y visuales. El primero es bucal, centrado en el propio cuerpo, se abre con la manipulación de 
objetos y se extiende con la aparición de la marcha. El espacio topológico se caracteriza por las relaciones concretas de cercanía, orden y separación. Espacio proyectivo donde los ejes y las relaciones espaciales serán respetadas. Espacio euclidiano que es la elaboración interiorizada del espacio, el espacio vivido se reelabora en el nivel de la representación.

5. Elaboración del Tiempo: es un proceso semejante al de la construcción del espacio, al inicio existe un tiempo vivido ligado al sueño y la vigilia, al hambre y la comida, al organismo y a la acción concreta, por lo que existen tantos tiempos como acciones. Estos hechos, que se perciben por medio de los cambios, forman los elementos básicos para la elaboración del tiempo. Con la función simbólica se integra la temporalidad, el niño se sitúa en el ahora, y a partir de éste, en un antes o un después, le permite distinguir situaciones simultáneas y sucesivas. Un hecho importante en la elaboración temporal es la percepción del cambio. La sucesión de hechos tiene su ritmo en el que se puede distinguir dos aspectos principales, que son complementarios y que contrastan entre sí: a. El ritmo interior que es orgánico, fisiológico, como la respiración. b. El ritmo exterior como el día y la noche, los acontecimientos observados en la vida cotidiana. Estos aspectos tienen una influencia recíproca, el ritmo participa en la elaboración de los movimientos, especialmente en la adquisición de los automatismos.

\section{Educación Motriz}

Esta se define como la educación general basada en la actividad corporal y que desarrolla los aspectos fundamentales de las capacidades motrices en relación con el desarrollo evolutivo en general.

Este término surge debido a la controversia que existe en relación a que la psicomotricidad concibe al movimiento como un fin, mientras que para la educación motriz esta se considera como un medio. La educación motriz es un tipo de aprendizaje, que pretende alcanzar un movimiento nuevo, aprehenderlo, consolidarlo y posteriormente aplicarlo o bien mejorar el que ya tiene. Para este autor los factores que intervienen en la conducta motora son: "el cuerpo, que se mueve, el espacio, donde se mueve, el tiempo cuando se mueve y las relaciones con que se mueve".

La Educación Motriz, permite que el niño pueda buscar nuevas actividades de movimiento, con lo cual amplía aquellos que ya posee y con ello fomentar las actitudes de descubrir y crear. La educación motriz se centra en capacitar a los niños y niñas a que puedan enfrentarse a sí mismo y al ambiente en el que se desenvuelven, para dar respuesta adecuada a las situaciones que se le presenten.

La Educación Motriz es la base metodológica de la Educación Física, la cual se desarrolla en el transcurso de la vida escolar, pero debe de estimularse desde el inicio de la fase del proceso educativo, es por ello que debe dividirse en dos fases: la primera de los 4 a los 6 años y la segunda de los 7 a los 9 años, y ésta debe de centrarse en el desarrollo coordinativo motor.

En la primera fase se debe propiciar la conciencia corporal, la adecuada estructuración y la organización del espacio-temporo-objetal. Y la segunda como el adecuado desarrollo de las 
capacidades de la coordinación general (gruesa) y específica (fina). La Educación Motriz no difiere mucho de la clasificación que hacen la Estimulación Temprana y la Psicomotricidad, todas dividen el desarrollo motor por etapas, para la educación motriz la primera etapa comprende:

1. Desarrollo Motor en el primer año de vida:

a) Crecimiento céfalo caudal: los movimientos son controlados de la cabeza hacía los pies.

b) Desarrollo de los movimientos contra laterales: son los movimientos que se realizan por una parte del cuerpo, conjuntamente con la parte situada al lado contrario.

c) Desarrollo de la Prensión: consiste en cerrar fuertemente la mano cuando le es colocado un objeto en ella.

d) Alcance de la Posición estática: consiste en el intento por ir poniéndose de pie, para ello se va colocando en diferentes posiciones hasta lograrlo.

e) Desarrollo de la locomoción: los intentos por trasladarse de un lugar a otro, para esto puede arrastrarse, gatear o caminar con o sin ayuda.

2. El desarrollo Motor de los Párvulos (1 a 6 años): Esta etapa del desarrollo se distingue porque los cambios se presentan en una forma rápida, el niño es distraído y le cuesta atender las indicaciones, dentro de las características de esta etapa están:

a) Caminar: el niño se traslada de un lugar a otro, puede realizar movimientos diferentes con sus brazos.

b) Trepar: consiste en poder "subirse" a diferentes objetos a determinadas alturas y en diferentes posiciones.

c) Escalar: subir y bajar escaleras al inicio a cuatro a manos y posteriormente con ayuda de otra persona o sosteniéndose pasamano o la pared.

d) Correr: se traslada de un lugar a otro en forma más rápida, va desarrollando un ritmo entre los movimientos de las piernas, los brazos y otras partes del cuerpo.

e) Saltar: al inicio lo hace de arriba hacia abajo, después puede hacerlo, al contrario, aprende también a saltar sobre un pie o sobre los dos, en forma estática o tomando distancia.

f) Lanzar: el niño aprende a "tirar" al inicio pequeños objetos con ambas manos, luego va desarrollando la capacidad de hacerlo con una mano, puede también obtener la habilidad de lanzar hacía un objetivo específico.

g) Atrapar: al inicio atrapa todo aquello que se le lanza a las manos, estando cerca de él y con poca fuerza. Posteriormente logra decepcionar el objeto utilizando sus manos, las cuales va a colocar en dirección al objeto que se le lanza.

h) Combinación de correr y saltar: el niño corre y salta los obstáculos que se le pongan al frente.

i) Combinación de correr y lanzar: al inicio el movimiento de lanzar se realiza en forma estática, después de los 6 años el niño o la niña ya puede desarrollar esta habilidad.

j) Combinación de correr y patear: se presenta a los 4 años, los niños y las niñas pueden patear cualquier objeto situado a una distancia de ellos.

k) Combinación de atrapar y lanzar: el niño puede atrapar los objetos en el aire y luego lanzarlos, esto es a los 6 años de edad. 
Combinación de lanzar y atrapar: el niño puede lanzar cualquier objeto al aire y luego puede atraparlo. Él logra de estas actividades por los niños y niñas dependerá mucho del estímulo que tengan dentro del hogar, la escuela y el ambiente donde se desenvuelven. Desarrollo Motor en los primeros años escolares: Todos los estímulos externos, todas las percepciones, todas las impresiones, los traducen los niños en movimiento y basta el más mínimo estímulo para impulsar esta movilidad.

Los niños y niñas a esta edad ya pueden concentrarse y prestar atención para la realización de actividades más precisas, algunas veces es difícil que se distraigan por otro tipo de situaciones. Las características de esta edad, en lo que a movimiento se refiere son las siguientes:

a) Correr: lo hacen en una forma más coordinada, regularmente pasan la mayor parte del tiempo haciéndolo.

b) Saltar: lo práctica constantemente, lo hacen hacía arriba o hacia delante con facilidad y destreza.

c) Lanzar: dependiendo del tamaño del objeto, regularmente lo hará con ambas manos o con una mano si la dimensión del objeto es menor.

d) Atrapar: puede hacerlo cuando el mismo lanza la pelota al aire o cuando otra persona lo hace, puede que en esta etapa aún presente cierto temor de golpearse o ser golpeado.

e) Combinaciones de movimiento: en esta edad lo hará en una forma más coordinada, haciéndolo de tal manera que pueda anticiparse al movimiento siguiente.

\section{Justificación}

La importancia de la escritura se ha podido observar a lo largo de toda la historia del hombre. Para ello remontamos al final de etapa de la Prehistoria, donde los humanos se comunicaban a través de dibujos (arte rupestre). A medida que fueron evolucionando surgió la necesidad de comunicarse por un medio escrito, en el que quedara plasmado aquello que consideraban importante (los nombres de las personas que formaban los grupos-tribus, la cantidad de cosecha recolectada...). Así, surgió la escritura, en el año 3000 a.C en Mesopotamia, dando comienzo a una de las importantes etapas de la historia de la humanidad, el fin de la Prehistoria y comienzo de la Historia. Existe una gran controversia en cuanto a cuándo se debe enseñar a los niños a leer y escribir. Si nos regimos por la legislación vigente, cuando el alumno comienza la etapa de Educación Infantil, no aparece la lectoescritura como uno de los objetivos mínimos que el alumno debe alcanzar. Sin embargo, desde la experiencia, en todos los colegios en los que he trabajado, la mayoría de los niños finalizan la etapa de Ed. Infantil sabiendo leer y escribir. El motivo que me ha llevado a elegir el presente tema para realizar este TFG radica en lo imprescindible que resulta la lectoescritura para asentar las bases en la adquisición de otros contenidos a trabajar y conseguir por el alumnado. Como docente, y tras la experiencia diaria, puedo decir que muchas de las

La lectoescritura en Educación Primaria Vanesa Lucas Griñán 8 dificultades que surgen en el alumnado, como la falta de comprensión lectora, ortografía, resolución de problemas... se deben a una carencia del hábito lector, de no valorar la lectura, ya no como herramienta para el aprendizaje, sino también como medio de ocio y diversión. Por ello, con este trabajo de investigación, pretendo plasmar una visión sobre el proceso de enseñanza-aprendizaje de la lectoescritura, qué métodos resultan más útiles, a qué se deben los problemas que surgen, y, sobre todo, con el fin de mejorar nuestra práctica docente 


\section{Fundamentación teórica}

1. ¿Qué es la lectura?

Existen multitud de definiciones a cerca de la lectura. La Real Academia de la Lengua Española (RAE) define la lectura como la acción de leer, la cual consiste en pasar la vista por un escrito o impreso comprendiendo la significación de los caracteres empleados. Sin embargo, existen diferentes definiciones que afirman que la lectura es conjunto de habilidades y capacidades, tratándose de un proceso de transacción entre el lector y el texto.

Podríamos escribir cientos de definiciones a cerca de la lectura, pero nos centraremos en la pedagoga $\mathrm{M}^{\mathrm{a}}$ Eugenia Dubois citada por (Lucas, 2014) la cual define: "la lectura es el producto de la interacción entre el pensamiento y el lenguaje". Dubois, distingue varios niveles en la comprensión lectora:

Nivel 1: comprender lo que está escrito en el texto de forma explícita.

Nivel 2: comprender lo que está escrito en el texto de forma implícita.

Nivel 3: hacer una lectura crítica y o tener la capacidad para evaluar la calidad del texto, ideas y propósito del autor.

\section{Métodos de enseñanza de la lectura y escritura}

En los métodos para la enseñanza de la lectura y escritura se marcan diversos conceptos y metodologías. Los métodos tradicionales plantean que para el logro de la lectura y escritura el niño debe alcanzar la habilidad de decodificar los elementos que conforman el texto escrito y después descifrar el significado o contenido. No existe un método preciso y eficaz, ya que cada niño posee características personales distintas. Siguiendo a Berta Braslavsky (2005) distinguimos los siguientes métodos tradicionales:

\section{Método Sintético}

Parten de la unidad más pequeña (letras o sílabas) a la más compleja (palabra), según señala (Sepúlveda, 2012) parten de lo más abstracto para llegar a lo concreto. Se basa en que los componentes de las palabras (letras y sílabas), constituyen un pilar indispensable para lectura y la escritura, se inicia con la enseñanza de estos elementos para combinarlos en formas lingüísticas de mayor complejidad. Estos métodos han sido denominados como métodos de la escuela tradicional, lo cual han sido muy criticados, lo que ha creado el método alfabético.

Este se basaría en:

- Adquisición del abecedario.

- Asociación de los grafemas con sus nombres. Ejemplo: a, be, ce, de, efe...

- Combinación de grafemas en sílabas (deletreo). Ejemplo: ene-a= na

- Combinar sílabas en palabras. Ejemplo: ese-o=so, ele-a=la, sola

\section{Método silábico}

Consiste en la enseñanza de las vocales. (Lucas, 2014) lo define como "el proceso mediante el cual se enseña la lectoescritura y consiste en la enseñanza de las vocales". Posteriormente la enseñanza de las consonantes se va combinando con las vocales formando sílabas y luego palabras. 
3. Método fonético(fonológico)

Se fundamente en la enseñanza de los fonemas o sonido distintivos de las letras.

La metodología del aprendizaje de la lectura con este método sería:

- Requiere del análisis y discriminación visual y fonológica de los grafemas y fonemas.

- El orden de presentación de los fonemas es muy relevante debido a los contrastes auditivos semejantes de algunos fonemas, por lo que resultan fundamentales los criterios de agrupación.

- Lectura y escritura de sílabas compuesta directas (vocal + consonante) e inversas (consonante + vocal).

- Lectura y escritura de palabras y frases compuestas por sílabas directas e inversas.

- Lectura y escritura de sílabas, palabras y frases trabadas.

- La lectura se va aprendiendo de manera simultánea con la escritura.

4. Método Analítico

Este método es atribuido a Ovidio Decroly, llamado método global. Parten de unidades mayores, concretas, como son las frases o las palabras, para llegar más adelante a las unidades más pequeñas y abstractas (sílabas y letras). Se debe iniciar con la frase o expresiones significativas para luego llegar a los componentes, es decir a las sílabas y las letras.

5. Métodos mixtos o eclécticos

Se centran en que el reconocimiento de palabras, la comprensión y el descubrimiento de las correspondencias grafema-fonema se produzcan de forma combinada y se realimenten entre sí. Al respecto, (Lucas, 2014) plantea: "Son procedimientos de análisis y síntesis, de discriminación de estímulos visuales simples y de estímulos visuales complejos que parecen aprenderse de manera simultánea e indisoluble. El deletreo conduce a la lectura de palabra y la lectura de palabras implica deletreo".

\section{Conclusión}

No cabe ninguna duda de que los tiempos y las personas cambian, tal como nos lo recuerda Lewis Carroll en Alicia en el país de las maravillas, cuando la protagonista le asegura a una oruga: por lo menos sé quién era yo cuando me levanté esta mañana, pero me parece que debo haber cambiado varias veces desde entonces». En efecto, porque la capacidad de transformación es una característica del ser humano, es que los docentes no podemos empeñarnos en reproducir modelos didácticos del pasado que, por obsoletos, estarían fuera del contexto socio-educativo actual.

\section{Bibliografía}

Blogger. (3 de Noviembre de 2008). http://desarrolloadulteztardia.blogspot.com/2008/11/desarrollopsicosocial.html. Obtenido de http://desarrolloadulteztardia.blogspot.com/2008/11/desarrollopsicosocial.html:

http://desarrolloadulteztardia.blogspot.com/2008/11/desarrollopsicosocial.html

Boletín Oficial Español . (2006 ). Ley Orgánica 2/ 2006 de 3 de mayo, de Educación . Madrid : Legislación Consolidada. 
Dr. C. Eddy Betancourt Escobar1, MSc. Jorge Alberto Rassa Parra2. (14 de Junio de 2016|). Inclusión Educativa Para Personas Con Necesidad Especiales En Las Universidades. Ponencia. Guayaquil, Guayas, Ecuador: Inexada- UIDE.

Exposito, F. (05 de 10 de 2011). Mente y Cerebro. Obtenido de Violencia de Genero : http://www.investigacionyciencia.es/files/7283.pdf

Flores Santiago, V. H. (27 de Enero de 2014). https://es.slideshare.net/vichuflosan/el-empirismo30501191. (V. H. Flores Santiago, Ed.) Recuperado el 19 de Enero de 2018, de es.slideshare.net/: https://es.slideshare.net/vichuflosan/el-empirismo-30501191

Friedan, B. (24 de Mayo de 2011). http://es.slideshare.net/BELLOTAH/desarrollo-psicosocial-en-laedad-adulta-tardia. Obtenido de http://es.slideshare.net/BELLOTAH/desarrollo-psicosocialen-la-edad-adulta-tardia: http://es.slideshare.net/BELLOTAH/desarrollo-psicosocial-en-laedad-adulta-tardia

Instituto Superior Pedagógico "José Martí",. (05 de Abril de 2004). http://www.bvs.sld.cu/revistas/revistahm/numeros/2004/n10/art/catedra.htm\#_ednref10.

Recuperado el 23 de 07 de 2015, de Adriana Guanche Martinez: http://www.bvs.sld.cu/revistas/revistahm/numeros/2004/n10/art/catedra.htm\#_ednref10

Lucas, V. (2014). La lectoescritura en la etapa de Educación Primaria. Soria: Universidad de Valladolid .

Miganjos, J. (2005). La importancia de la Educación motriz en el proceso de enseñanza de la lectoescritura en niños y niñas del nivel preprimario y de primero de primaria en escuelas oficiales del municipio de Quetzaltenango. Guatemala : Universidad Rafael Alcívar .

MSc. Zila Esteves Fajardo MSc. Ericka Figueroa. MSc. Norma Garces. (14 de Junio de 2016). Ponencia. Obtenido de Educación Inclusiva A Madres Y Padres Solteros Como Inversión Estatal Para El Desarrollo Sostenible De La Familia: http://www.slideshare.net/zilaiestevesfajardo

Sepúlveda, G. (2012). La Psicomotricidad fina y su importancia para la adquisición de la lectoescritura . Zamora- Michoacán : Biblioteca Nacional .

Sierra, C. P. (2017). Emprendimiento. España: Trillas.

Vivanco, M. E. (26 de julio de 2012). http://desarrolloadulteztardia.blogspot.com/2008/11/desarrollopsicosocial.html. Obtenido de http://desarrolloadulteztardia.blogspot.com/2008/11/desarrollopsicosocial.html: $\quad$ http://desarrolloadulteztardia.blogspot.com/2008/11/desarrollopsicosocial.html

Wallon, H. (10 de Octubre de (1879- 1962)). Aprendizaje y Desarrollo Motor E.F. Obtenido de Teoria de Henri Wallon: http://aprendizajeydesarrollomotoref.blogspot.com/2015/10/modelos-deldesarrollo-motor.html 